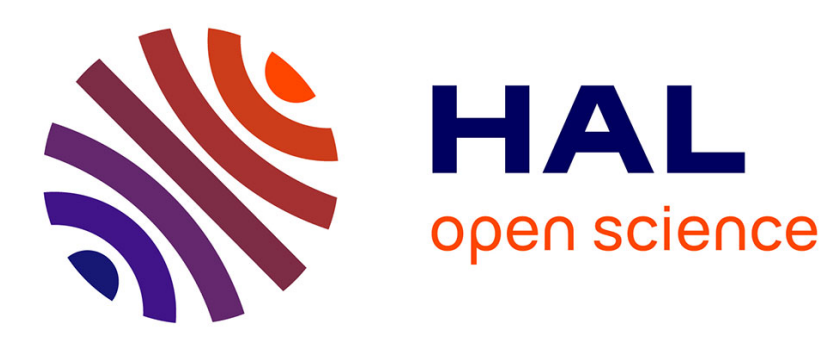

\title{
Lower bound static approach for the yield design of thick plates
}

Jérémy Bleyer, Patrick de Buhan

\section{To cite this version:}

Jérémy Bleyer, Patrick de Buhan. Lower bound static approach for the yield design of thick plates. International Journal for Numerical Methods in Engineering, 2014, 100 (11), pp.814-833. 10.1002/nme.4776 . hal-01082202

\section{HAL Id: hal-01082202 \\ https://hal.science/hal-01082202}

Submitted on 15 Nov 2014

HAL is a multi-disciplinary open access archive for the deposit and dissemination of scientific research documents, whether they are published or not. The documents may come from teaching and research institutions in France or abroad, or from public or private research centers.
L'archive ouverte pluridisciplinaire HAL, est destinée au dépôt et à la diffusion de documents scientifiques de niveau recherche, publiés ou non, émanant des établissements d'enseignement et de recherche français ou étrangers, des laboratoires publics ou privés. 


\title{
Lower bound static approach for the yield design of thick plates
}

\author{
Jeremy Bleyer ${ }^{\mathrm{a}, *}$, Patrick de Buhan ${ }^{\mathrm{a}}$ \\ ${ }^{a}$ Université Paris-Est, Laboratoire Navier, \\ Ecole des Ponts ParisTech-IFSTTAR-CNRS (UMR 8205) \\ 6-8 av Blaise Pascal, Cité Descartes, 77455 Champs-sur-Marne, FRANCE
}

\begin{abstract}
The present work addresses the lower bound limit analysis (or yield design) of thick plates under shear-bending interaction. Equilibrium finite elements are used to discretize the bending moment and the shear force fields. Different strength criteria, formulated in the fivedimensional space of bending moment and shear force, are considered, one of them taking into account the interaction between bending and shear resistances. The criteria are chosen to be sufficiently simple so that the resulting optimization problem can be formulated as a second-order cone programming problem, which is solved by the dedicated solver MosEK. The efficiency of the proposed finite element is illustrated by means of numerical examples on different plate geometries, for which the thin plate solutions as well as the pure shear solutions are accurately obtained as two different limit cases of the plate slenderness ratio. In particular, the proposed element exhibits a good behavior in the thin plate limit.
\end{abstract}

Keywords: limit analysis, yield design, lower bound, thick plates, finite element method, second order cone programming

\section{Introduction}

Due to the development of efficient mathematical programming solvers, numerical yield design (or limit analysis in the specific case of a rigid perfectly plastic behavior) has gained increasing attention in the last decades. The resolution of a yield design problem can be formulated using two different approaches. The first one, namely the static approach, consists of maximizing the loading factor over a set of stress fields which are statically admissible and fulfill the strength criterion at each point of the structure. The second approach, namely the kinematic approach, is only the mathematical dualization of the first one, by means of the virtual work principle. It leads to the minimization of a convex function, the maximum resisting work, over the set of kinematically admissible virtual velocity fields and under a normalization condition of the power of external loads. The finite element method has been

\footnotetext{
* Correspondence to: J. Bleyer, Laboratoire Navier, 6-8 av Blaise Pascal, Cité Descartes, 77455 Champssur-Marne, France, Tel : +33 (0)1641536 59

Email address: jeremy.bleyer@enpc.fr (Jeremy Bleyer)

$U R L:$ https://sites.google.com/site/bleyerjeremy/ (Jeremy Bleyer)
} 
used for both approaches : the stress fields and associated equilibrium equations being discretized in the static approach [1,2], whereas the virtual velocity fields are discretized to compute the maximum resisting work in the kinematic approach $[3,4]$.

Yield design theory being a general framework for estimating the ultimate load of any structure, it has also been formulated in the specific case of plates and slabs [5-8]. Most of the work in this area has been devoted to the case of thin plates in bending, i.e. plates possessing a finite resistance to bending moments only, the resistance to shear and membrane forces being assumed to be infinite. One famous method to estimate the collapse load of such structures is the yield line theory developed by Johansen [5], which is a kinematic approach involving rigid failure mechanisms separated by yield lines. Finite element method has also been proposed to solve kinematic approaches for thin plates in bending, either as a numerical implementation of the yield line theory [9] or by considering more complex velocity fields [10-13]. Similarly, finite elements discretizing the thin plate equilibrium equations have also been proposed to solve the static approach [12, 14, 15].

However, to the author's knowledge, little work has been devoted to the case of "thick" plates for which a limited shear resistance has to be taken into account. The work of Cecchi and co-workers [16] on masonry walls for instance considered a Reissner-Mindlin kinematic limit analysis finite element, in which dissipation is produced at the elements edges due to bending moment, torsion and shear. In [11] and [17], quadrilateral finite elements have been proposed to solve the kinematic approach for thick plates. It is worth noting that, without any specific treatment, these elements exhibit shear-locking in the thin plate limit. To overcome this effect, the authors proposed a mixed B-bar strain formulation. Very recently, Canh V. Le [18] proposed to use a discrete shear gap stabilization technique to overcome shear locking, still for the kinematic approach.

It seems that, so far, no finite element has been proposed to solve the static approach for thick plates.

Following the point of view adopted in $[12,15]$, the present formulation only relies on a plate model, in the sense that it does not refer to any corresponding three dimensional structure. The only optimization variables will, therefore, be the bending moment and the shear force fields throughout the whole plate. Besides, one key aspect of the static approach relies in the definition of the strength criterion. Hence, in this work, strength criteria will have to be formulated in the five-dimensional space of bending moments and shear forces. In order to keep computations tractable, simple generalized strength criteria will be considered.

The formulation of the yield design thick plate problem will be the purpose of Section 2. Section 3 is devoted to the finite element discretization of the problem and mathematical programming aspects. Some considerations on the shear-locking effect are then given in Section 4. Finally, Section 5 is concerned with numerical examples which are used to validate the proposed formulation. 


\section{Yield design of thick plates}

\subsection{Description of statically admissible fields}

Let $\Omega$ be a plate of thickness $t$ occupying the plane $(O x y)$ in a cartesian frame $\left(\underline{e}_{x}, \underline{e}_{y}, \underline{e}_{z}\right)$. The internal forces of the plate are described by a symmetric tensor $\underline{N}$ of membrane forces, a symmetric tensor $\underline{\underline{M}}$ of bending moments and a vector $\underline{V}$ of shear forces. The external forces are represented by a transversal distributed pressure $f_{z}=-p_{D}$ and, possibly, loads distributed along a line $\Gamma$ with a transversal component $F_{z}=-P_{L}$. Distributed exterior couples and in-plane loads can also be considered in this model, but will be taken to zero in the following for the sake of simplicity. Note that the sign convention for bending moments is such that positive bending corresponds to traction of the bottom face of the plate.

With the previous notations, the local equations of equilibrium in this model are given by :

$$
\begin{aligned}
\operatorname{div} \underline{\underline{N}} & =\underline{0} \\
\operatorname{div} \underline{V}-p_{D} & =0 \\
\operatorname{div} \underline{M}+\underline{V} & =\underline{0}
\end{aligned}
$$

whereas the jump equations through a line $\Gamma$ of normal $\underline{n}$ read as :

$$
\begin{aligned}
& \llbracket \underline{\underline{N}} \rrbracket \cdot \underline{n}=\underline{0} \\
& \llbracket \underline{V \rrbracket} \underline{n}-P_{L}=0 \\
& \llbracket \underline{\underline{M}} \rrbracket \cdot \underline{n}=\underline{0}
\end{aligned}
$$

where $\llbracket \cdot \rrbracket$ denotes the jump of the quantity $\cdot$ through the line $\Gamma$ following its unit normal $\underline{n}$. Finally, the boundary conditions on the plate boundary $\partial \Omega$ of normal $\underline{n}$ are written by prescribing (or not) some components of the in-plane reaction $\underline{T}=\underline{\underline{N}} \cdot \underline{n}$, the vertical reaction $R=\underline{V} \cdot \underline{n}$ or the resulting bending moment $\underline{\mathcal{M}}=\underline{\underline{M}} \cdot \underline{n}$.

A set of fields $(\underline{N}, \underline{V}, \underline{M})$ will then be said to be statically admissible with a set of external forces $\left(p_{D}, P_{L}\right)$ if they satisfy the local equilibrium equations (1)-(3), the jump equations (4)-(6) and the prescribed boundary conditions.

\subsection{Yield criteria for thick plates in the $(\underline{\underline{M}}, \underline{V})$ space}

Generally speaking, the strength criterion of a plate can be formulated as a condition involving all internal forces (generalized stresses) at any point of the plate, namely :

$$
f(\underline{\underline{N}}, \underline{V}, \underline{\underline{M}}) \leq 0
$$

The present work aims at investigating the resistance of bending plates when the plate cannot be really considered as thin. It will therefore be assumed that the plate is infinitely 


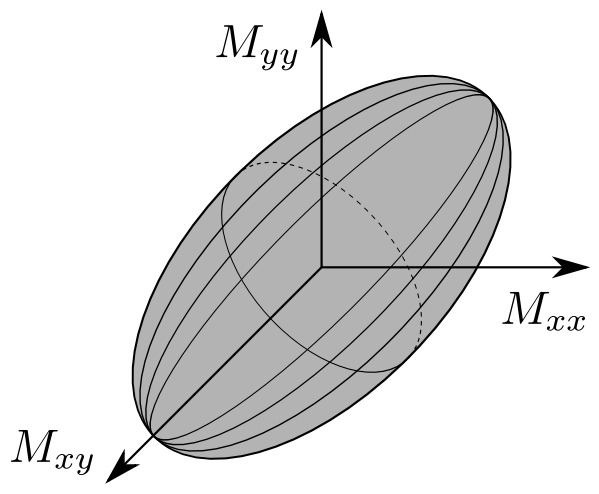

(a) Representation of the von Mises bending criterion $G_{M}$

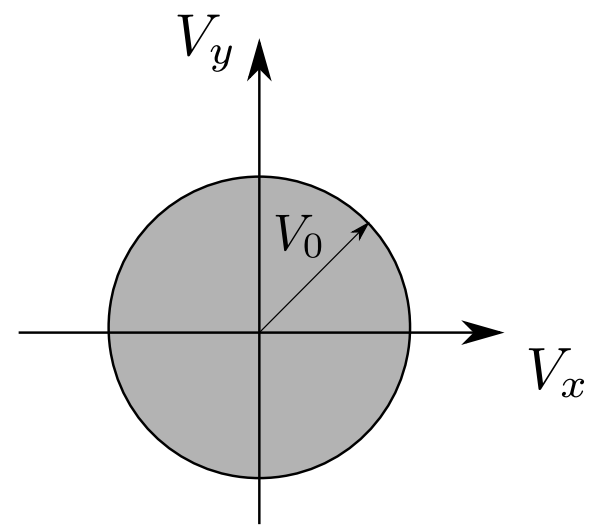

(b) Representation of the shear criterion $G_{V}$

Figure 1: Bending-only and shear-only strength criteria $G_{M}$ and $G_{V}$

resistant to membrane forces, but not to bending moment and shear forces so that the strength criterion reduces to :

$$
f(\underline{V}, \underline{\underline{M}}) \leq 0
$$

The strength domain in the five-dimensional space will be defined as :

$$
G=\{(\underline{\underline{V}}, \underline{\underline{M}}) \text { s.t. } f(\underline{V}, \underline{\underline{M}}) \leq 0\}
$$

In the following, the intersection of the strength domain $G$ with the subspace of zero shear forces $\underline{V}=\underline{0}$, will be noted $G_{M} \subset \mathbb{R}^{3}$ and taken as the classical von Mises ellipsoid criterion for bending plates :

$$
G_{M}=\left\{\underline{\underline{M}} \text { s.t. } f_{M}(\underline{\underline{M}}) \leq 0\right\}
$$

with

$$
f_{M}(\underline{\underline{M}})=\sqrt{M_{x x}^{2}+M_{y y}^{2}-M_{x x} M_{y y}+3 M_{x y}^{2}}-M_{0}=\|\underline{\underline{M}}\|_{v M}-M_{0}
$$

Similarly, the intersection of the strength domain with the subspace of zero bending moments $\underline{\underline{M}}=\underline{\underline{0}}$, will be noted $G_{V} \subset \mathbb{R}^{2}$ and taken as a simple criterion limiting the norm of the $\overline{\overline{\text { shear }}} \overline{\overline{\text { force vector : }}}$

$$
G_{V}=\left\{\underline{V} \text { s.t. } f_{V}(\underline{V}) \leq 0\right\}
$$

with

$$
f_{V}(\underline{V})=\sqrt{V_{x}^{2}+V_{y}^{2}}-V_{0}=\|\underline{V}\|-V_{0}
$$

The ultimate bending moment $M_{0}$ and shear force $V_{0}$ are material parameters describing the plate strength to pure bending and pure shear respectively. Referring to a threedimensional modelling of the plate of thickness $t$, associated with a von Mises strength 
criterion of ultimate tensile/compressive strength $\sigma_{0}$, then, $M_{0}$ and $V_{0}$ are given by the following relations :

$$
M_{0}=\frac{\sigma_{0} t^{2}}{4} \quad ; \quad V_{0}=\frac{\sigma_{0} t}{\sqrt{3}}
$$

In the following applications, three different forms will be considered for the complete strength criterion associated to $G$ (figure 2) :

1. infinitely resistant shear forces : $G=\left\{(\underline{V}, \underline{\underline{M}}) \in \mathbb{R}^{5}\right.$ s.t. $\left.\underline{\underline{M}} \in G_{M}\right\}=\mathbb{R}^{2} \times G_{M}$

2. no shear-bending interaction : $G=\left\{(\underline{\underline{V}}, \underline{\underline{M}}) \in \mathbb{R}^{5}\right.$ s.t. $\underline{V} \overline{\bar{\in}} G_{V}$ and $\left.\underline{\underline{M}} \in G_{M}\right\}=G_{V} \times$ $G_{M}$

3. shear-bending interaction : $G=\left\{(\underline{\underline{V}}, \underline{\underline{M}}) \in \mathbb{R}^{5}\right.$ s.t. $\left.f_{V M}(\underline{V}, \underline{\underline{M}}) \leq 0\right\}$ with :

$$
f_{V M}(\underline{V}, \underline{\underline{M}})=\sqrt{\left(\frac{\|\underline{\underline{M}}\|_{v M}}{M_{0}}\right)^{2}+\left(\frac{\|\underline{V}\|}{V_{0}}\right)^{2}}-1
$$

It is worth noting that the first criterion with infinitely resistant shear forces can be obtained as the limit of the two other criteria when $V_{0} L / M_{0} \rightarrow \infty$, where $L$ is a characteristic length of the plate. On account of (7), it corresponds to the limit $\beta=L / t \rightarrow \infty, \beta$ being the slenderness ratio of the plate. Hence, the first criterion corresponds to a thin plate limit of the two other criteria.

The second criterion is obtained as the product of $G_{M}$ and $G_{V}$, so that there is no interaction between shear and bending strength capacities. On the contrary, the third criterion can be represented as an ellipsoid in $\mathbb{R}^{5}$, the intersection of which with the subspace $\underline{V}=\underline{0}$ is represented by $G_{M}$ whereas its intersection with the subspace $\underline{M}=\underline{0}$ is represented by $G_{V}$. Hence, the criterion with interaction is strictly contained in the second criterion with no interaction, which is itself contained in the criterion with infinite shear strength. It is to be noted that the interaction criterion, associated with expressions (7), has already been proposed in previous works $[19,20]$ and can be seen as an extension to plates of the strength criterion for idealized I-beams under combined bending and shear.

Indeed, in the case of a local von Mises criterion, the expression of the support function (or plastic dissipation in the context of limit analysis) accounting for shear deformations can be expressed as [21] :

$$
\pi(\underline{\underline{\chi}}, \underline{\gamma})=\sigma_{0} \int_{-t / 2}^{t / 2} \sqrt{z^{2} \chi^{T} \mathbf{Q}_{b} \boldsymbol{\chi}+\gamma^{T} \mathbf{Q}_{s} \gamma} d z
$$

where $\chi=\left(\chi_{x x}, \chi_{y y}, 2 \chi_{x y}\right)^{T}$ is the vector in Voigt notation of the curvature rate tensor $\underline{\underline{\chi}}$, $\gamma=\left(\gamma_{x}, \gamma_{y}\right)^{T}$ the shear strain vector components, $t$ is the plate thickness and

$$
\mathbf{Q}_{b}=\frac{1}{3}\left[\begin{array}{lll}
4 & 2 & 0 \\
2 & 4 & 0 \\
0 & 0 & 1
\end{array}\right] \quad \mathbf{Q}_{s}=\frac{1}{3}\left[\begin{array}{ll}
1 & 0 \\
0 & 1
\end{array}\right]
$$

The exact strength criterion $G^{*}$ expressed in terms of the bending moment and shear force vector can be obtained from the expression of this support function as follows :

$$
(\underline{\underline{M}}, \underline{V}) \in G^{*} \Longleftrightarrow \underline{\underline{M}}: \underline{\underline{\chi}}_{5}+\underline{V} \cdot \underline{\gamma} \leq \pi(\underline{\underline{\chi}}, \underline{\gamma}) \quad \forall \underline{\underline{\chi}}, \underline{\gamma}
$$


a) infinite shear resistance

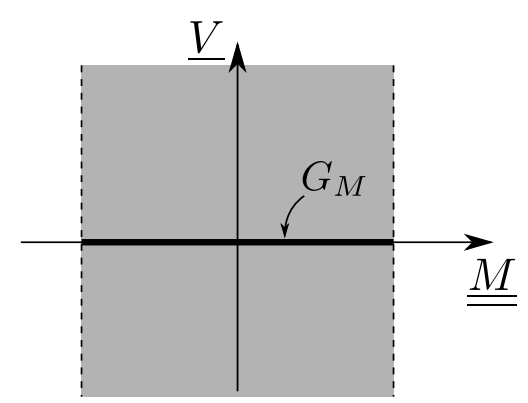

b) no interaction

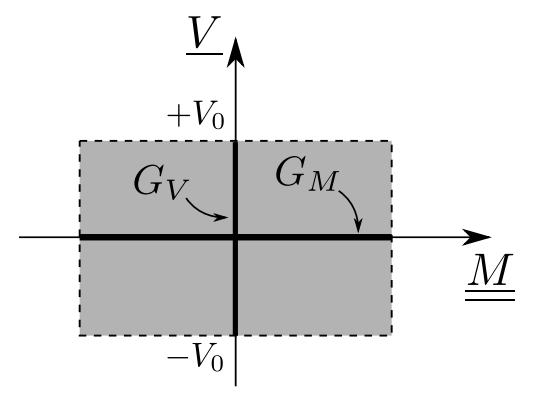

c) with interaction

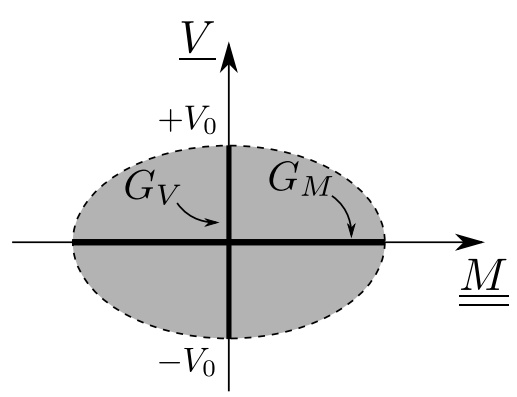

Figure 2: Schematic representation of the different strength criteria

As stated in [11], expression (9) is often approximated using a ng-points Gauss quadrature over the half thickness, so that :

$$
\pi(\underline{\underline{\chi}}, \underline{\gamma})=2 \sigma_{0} \int_{0}^{t / 2} \sqrt{z^{2} \boldsymbol{\chi}^{T} \mathbf{Q}_{b} \boldsymbol{\chi}+\boldsymbol{\gamma}^{T} \mathbf{Q}_{s} \boldsymbol{\gamma}} d z \approx \frac{1}{2} \sum_{g=1}^{n g} M_{0} \omega_{g} \sqrt{\left(1+\xi_{g}\right)^{2} \boldsymbol{\chi}^{T} \mathbf{Q}_{b} \boldsymbol{\chi}+\frac{16}{t^{2}} \boldsymbol{\gamma}^{T} \mathbf{Q}_{s} \boldsymbol{\gamma}}
$$

where $\xi=4 z / t-1$ and $\omega_{g}$ is the weighting factor of the Gauss point $\xi_{g}$. It is now quite easy to see that, in the case of a one-point integration rule $\left(\xi_{1}=0, \omega_{1}=2\right)$, equation (10) reduces to an approximate yield criterion given by :

$$
(\underline{\underline{M}}, \underline{V}) \in G^{a p p} \Longleftrightarrow \sqrt{\boldsymbol{M}^{T} \mathbf{P}_{b} \boldsymbol{M}+\frac{t^{2}}{16} \boldsymbol{V}^{T} \mathbf{P}_{s} \boldsymbol{V}}-M_{0} \leq 0
$$

where $\boldsymbol{M}=\left(M_{x x}, M_{y y}, M_{x y}\right)^{T}, \boldsymbol{V}=\left(V_{x}, V_{y}\right)^{T}$ and

$$
\mathbf{P}_{b}=\left[\begin{array}{ccc}
1 & -1 / 2 & 0 \\
-1 / 2 & 1 & 0 \\
0 & 0 & 3
\end{array}\right] \quad \mathbf{P}_{s}=3\left[\begin{array}{cc}
1 & 0 \\
0 & 1
\end{array}\right]
$$

which is exactly equivalent to the expression of the shear-bending interaction criterion (8). Therefore, this criterion can be seen as an upper-bound approximation of the exact strength criterion $G^{*}$ in the case of a plate made of a material obeying the von Mises criterion.

\subsection{Statement of the yield design problem for thick plates}

It is assumed from now on, that the plate loading depends upon several loading parameters collected in the vector $\underline{Q}$. In this context, the domain $K$ of potentially safe loads $\underline{Q}$, as introduced in the limit analysis or yield design theory [8], is defined as follows :

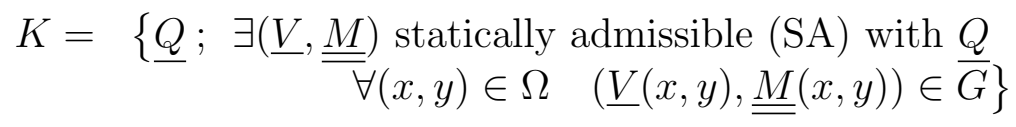



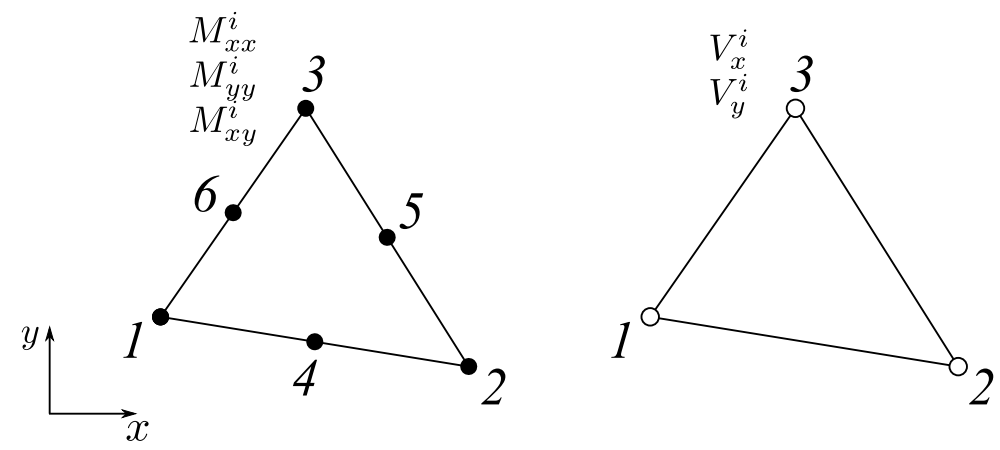

Figure 3: Degrees of freedom in a given element for the quadratic interpolation of $\underline{\underline{M}}$ and the linear interpolation of $\underline{V}$

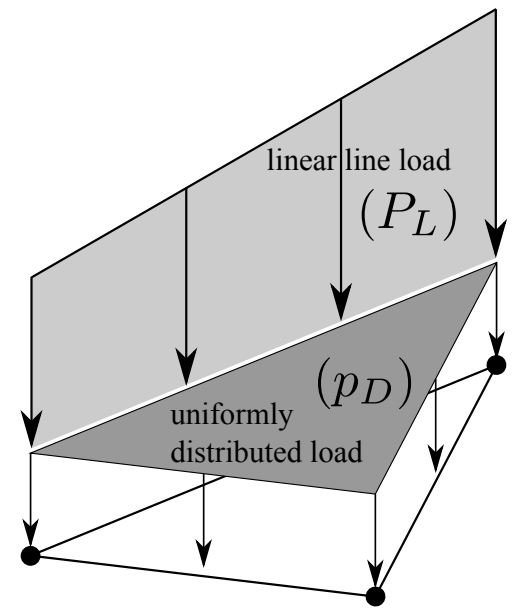

Figure 4: Admissible loads on a given element consistent with the chosen interpolations

\section{Finite element discretization}

\subsection{Equilibrium, jump equations and boundary conditions}

The numerical resolution of the previously mentioned static approach will be performed using a finite element discretization of the plate by considering piecewise continuous bending moment and shear force fields, separated by statically admissible discontinuities across adjoining elements. In the following, since the plate is assumed to be infinitely resistant to membrane forces, they will not be taken into account in the formulation and can be considered as reactions only.

In each triangular finite element $e$, a 6 -noded quadratic interpolation for each component of the bending moment tensor and a 3-noded linear interpolation of the shear vector will be assumed (figure 3). Hence, using classical linear $\left(N_{i}^{1}(\xi, \eta)\right)$ and quadratic $\left(N_{i}^{2}(\xi, \eta)\right)$ shape 
functions, we have in each element :

$$
\begin{aligned}
& \mathbf{V}(\xi, \eta)=\sum_{i=1}^{3} N_{i}^{1}(\xi, \eta) \mathbf{V}^{i} \\
& \mathbf{M}(\xi, \eta)=\sum_{i=1}^{6} N_{i}^{2}(\xi, \eta) \mathbf{M}^{i}
\end{aligned}
$$

where $\mathbf{M}=\left\langle\begin{array}{lll}M_{x x} & M_{y y} & M_{x y}\end{array}\right\rangle^{T}$ and $\mathbf{V}=\left\langle\begin{array}{ll}V_{x} & V_{y}\end{array}\right\rangle^{T}$.

Since the bending moment varies quadratically and the shear force varies linearly, the quantity $\operatorname{div} \underline{\underline{M}}+\underline{V}$ varies linearly in the element as well. It is, therefore, sufficient to enforce this quantity to be zero at the three end nodes of the triangle, so that equation (2) is satisfied exactly at every point of the element. Similarly, since $\operatorname{div} \underline{V}$ is constant in the element, only uniform distributed pressures in the element are possible for the chosen interpolation to satisfy equation (3) (figure 4). Then, in element $e$, the local equilibrium reads as :

$$
\left[\begin{array}{cc}
{\left[D_{M}^{1}\right]} & {\left[N_{V}^{1}\right]} \\
{\left[D_{M}^{2}\right]} & {\left[N_{V}^{2}\right]} \\
{\left[D_{M}^{3}\right]} & {\left[N_{V}^{3}\right]} \\
0 & {\left[D_{V}\right]}
\end{array}\right]\left\{\begin{array}{c}
\mathbf{M}^{1} \\
\vdots \\
\mathbf{M}^{6} \\
\mathbf{V}^{1} \\
\mathbf{V}^{2} \\
\mathbf{V}^{3}
\end{array}\right\}=\left\{\begin{array}{c}
0 \\
0 \\
\vdots \\
0 \\
p_{D, e}
\end{array}\right\}
$$

where $\left[D_{M}^{i}\right]\left(\right.$ resp. $\left.\left[N_{V}^{i}\right]\right)$ computes $\operatorname{div} \underline{\underline{M}}(\operatorname{resp} . \mathbf{V})$ at node $i$ and $\left[D_{V}\right]$ computes $\operatorname{div} \underline{V}$.

The jump equation for bending moments between two adjacent elements $e$ and $e^{\prime}$ has to be satisfied at all three nodes of the shared edge (figure 5). Let $i=1,2,3$ be a node in element $e$ and $i^{\prime}=1^{\prime}, 2^{\prime}, 3^{\prime}$ the corresponding node in element $e^{\prime}$, we have :

$$
\left[\begin{array}{cccccc}
{[b]} & 0 & 0 & -[b] & 0 & 0 \\
0 & {[b]} & 0 & 0 & -[b] & 0 \\
0 & 0 & {[b]} & 0 & 0 & -[b]
\end{array}\right]\left\{\begin{array}{l}
\mathbf{M}^{1} \\
\mathbf{M}^{2} \\
\mathbf{M}^{3} \\
\mathbf{M}^{1^{\prime}} \\
\mathbf{M}^{2^{\prime}} \\
\mathbf{M}^{3^{\prime}}
\end{array}\right\}=\left\{\begin{array}{c}
0 \\
\vdots \\
0
\end{array}\right\}
$$

with $[b]=\left[\begin{array}{ccc}n_{x}^{2} & n_{y}^{2} & 2 n_{x} n_{y} \\ n_{x} t_{x} & n_{y} t_{y} & n_{x} t_{y}+n_{y} t_{x}\end{array}\right], \underline{t}$ being the tangent unit vector (figure 5). The jump equation for shear forces has to be satisfied at the two end nodes of the shared edge only. With a linearly varying line load $P_{L}$ between nodes 1 and 2 , the jump equation reads as :

$$
\left[\begin{array}{cccc}
\langle\underline{n}\rangle & 0 & -\langle\underline{n}\rangle & 0 \\
0 & \langle\underline{n}\rangle & 0 & -\langle\underline{n}\rangle
\end{array}\right]\left\{\begin{array}{l}
\mathbf{V}^{1} \\
\mathbf{V}^{2} \\
\mathbf{V}^{1^{\prime}} \\
\mathbf{V}^{2^{\prime}}
\end{array}\right\}=\left\{\begin{array}{l}
P_{L}^{1} \\
P_{L}^{2}
\end{array}\right\}
$$




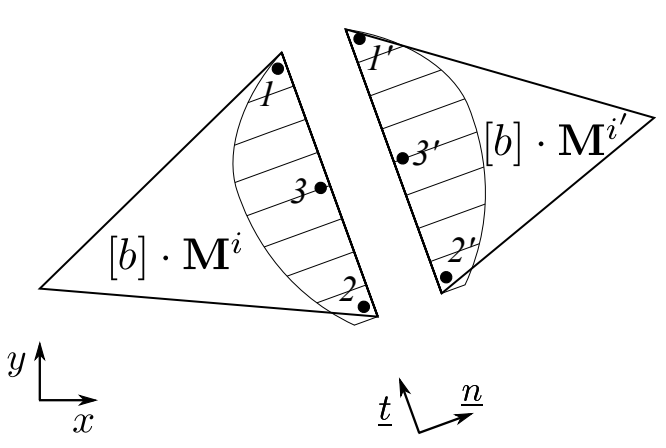

(a) Jump equation for $\underline{\underline{M}}$

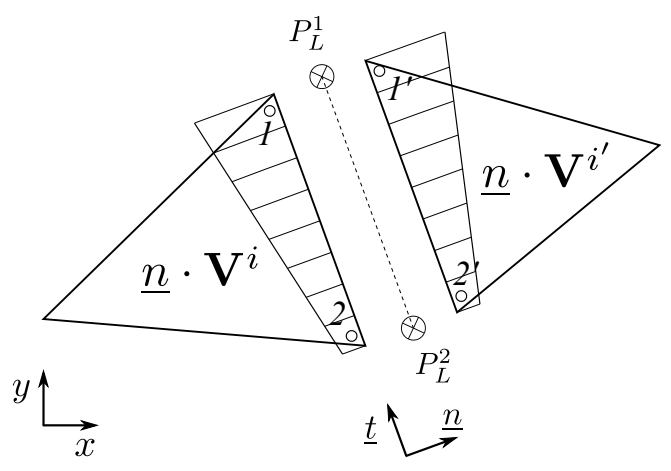

(b) Jump equation for $\underline{V}$

Figure 5: Satisfaction of the jump equations between adjacent elements

Boundary conditions are enforced exactly the same way by retaining the appropriate lines of the following equations (in the case of zero prescribed reactions) :

$$
\begin{gathered}
{\left[\begin{array}{ccc}
{[b]} & 0 & 0 \\
0 & {[b]} & 0 \\
0 & 0 & {[b]}
\end{array}\right]\left\{\begin{array}{l}
\mathbf{M}^{1} \\
\mathbf{M}^{2} \\
\mathbf{M}^{3}
\end{array}\right\}=\left\{\begin{array}{c}
0 \\
\vdots \\
0
\end{array}\right\}} \\
{\left[\begin{array}{cc}
\langle\underline{n}\rangle & 0 \\
0 & \langle\underline{n}\rangle
\end{array}\right]\left\{\begin{array}{l}
\mathbf{V}^{1} \\
\mathbf{V}^{2}
\end{array}\right\}=\left\{\begin{array}{l}
0 \\
0
\end{array}\right\}}
\end{gathered}
$$

All these elementary contributions of local equilibrium, jump equations and boundary conditions are then assembled into a global linear relationship between the vector of static unknowns $\boldsymbol{\Sigma}$ and an external force vector $\mathbf{F}$ of the form :

$$
\mathbf{H} \boldsymbol{\Sigma}=\mathbf{F}
$$

\subsection{Formulation of the strength criterion as conic constraints}

In order to obtain a rigorous lower bound, the strength criterion should be satisfied at each point inside a given element. Unfortunately, the quadratic variation of $\underline{\underline{M}}$ inside an element makes it impossible to determine a priori where the strength criterion maxima will occur. This issue has been circumvented in [12] by adopting a so-called criterion of the mean which corresponds to the satisfaction of the strength criterion only in a mean sense over each finite element.

In the following, it has been decided to check the strength criterion only at a finite number $N_{c}$ of points within the element, while keeping in mind that it can be violated between these points. It is always possible to imagine a post-processing procedure which will analyze the optimal static field to determine the exact position of the strength criterion maxima. If the criterion is violated at some of these points, a down-scaling factor can be applied to the ultimate load for the static field to fulfill the strength criterion exactly. Another idea would be to reoptimize separately the static fields on each element discretized into 


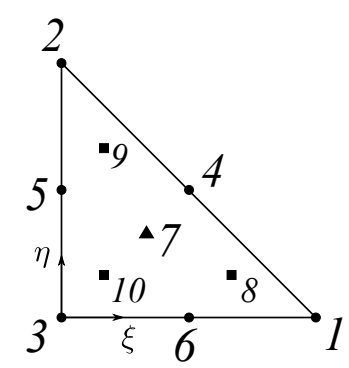

Figure 6: Position of the points used to check the criterion in a reference element up to $N_{c}=10$

\begin{tabular}{|c|c|c|}
\hline Point & $\xi$ & $\eta$ \\
\hline 1 & 1 & 0 \\
\hline 2 & 0 & 1 \\
\hline 3 & 0 & 0 \\
\hline 4 & $1 / 2$ & $1 / 2$ \\
\hline 5 & 0 & $1 / 2$ \\
\hline
\end{tabular} \mid \begin{tabular}{cc|c|c|}
\hline & Point & $\xi$ & $\eta$ \\
\hline 10 & $1 / 2$ & 0 \\
\hline
\end{tabular}

Table 1: Coordinates of the checking points in the reference triangle

many sub-elements using the approximate solution from the first computation as imposed boundary conditions on the element edges. However, it will be proved numerically that the error made on the ultimate load using a finite number of checking point is negligible and decreases when refining the mesh. The position of the checking points which will be used in the following are represented in figure 6 and given in table 1.

Now, let $c$ denote a checking point in a given element $e$. The von Mises criterion for bending moments and the strength criterion for shear forces only can be written at $c$ as :

$$
\begin{gathered}
f_{M}\left(\mathbf{M}^{c}\right)=\left\|\mathbf{J} \mathbf{M}^{c}\right\|-M_{0} \leq 0 \\
f_{V}\left(\mathbf{V}^{c}\right)=\left\|\mathbf{V}^{c}\right\|-V_{0} \leq 0
\end{gathered}
$$

where $\mathbf{J}=\left[\begin{array}{ccc}1 & -1 / 2 & 0 \\ 0 & \sqrt{3} / 2 & 0 \\ 0 & 0 & \sqrt{3}\end{array}\right]$

Therefore, introducing the following auxiliary variables $\mathbf{z}^{c}=\left\langle\frac{1}{M_{0}} \mathbf{J} \mathbf{M}^{c}, \frac{1}{V_{0}} \mathbf{V}^{c}\right\rangle^{T}$, the different strength criteria can be written as :

- infinite shear strength :

$$
\left\|\mathbf{z}_{1 \rightarrow 3}^{c}\right\| \leq 1 \quad \forall c=1, \ldots, N_{c} \cdot N_{E}
$$

- no interaction :

$$
\begin{aligned}
& \left\|\mathbf{z}_{1 \rightarrow 3}^{c}\right\| \leq 1 \\
& \left\|\mathbf{z}_{4 \rightarrow 5}^{c}\right\| \leq 1
\end{aligned} \quad \forall c=1, \ldots, N_{c} \cdot N_{E}
$$


- with interaction :

$$
\left\|\mathbf{z}^{c}\right\| \leq 1 \quad \forall c=1, \ldots, N_{c} \cdot N_{E}
$$

Clearly, in each case, the strength criterion can be formulated using second-order cone constraints of different sizes which can be summarized by a condition of the form $\mathbf{z}^{c} \in \mathcal{C}$. Finally, let us mention that for the second criterion with no shear-bending interaction, it is not necessary to satisfy the shear criterion at each checking points, but sufficient to fulfill it at the three nodes of the triangle since $\underline{V}$ varies linearly and $f_{V}$ is convex.

\subsection{Formulation of the yield design static approach as SOCP}

The global force vector is now assumed to be proportional to a reference load $\mathbf{F}_{\mathbf{0}}$ : $\mathbf{F}=\lambda \mathbf{F}_{\mathbf{0}}$. The resolution of the yield design static approach will give a lower bound $\lambda_{l} \leq \lambda^{+}$ to the exact ultimate load factor $\lambda^{+}$that the plate can sustain, on account of adopted strength criterion. This lower bound is obtained by solving the following maximization problem :

$$
\begin{aligned}
\lambda_{l}=\max & \lambda \\
\text { s.t. } & \mathbf{H} \boldsymbol{\Sigma}=\lambda \mathbf{F} \\
& \mathbf{A} \boldsymbol{\Sigma}=\left\langle\mathbf{z}^{1} \quad \ldots \quad \mathbf{z}^{N_{c} \cdot N_{E}}\right\rangle^{T} \\
& \mathbf{z}^{c} \in \mathcal{C} \quad \forall c=1, \ldots, N_{c} \cdot N_{E}
\end{aligned}
$$

where the auxiliary variables definitions have been assembled into the constraint $\mathbf{A} \boldsymbol{\Sigma}=$

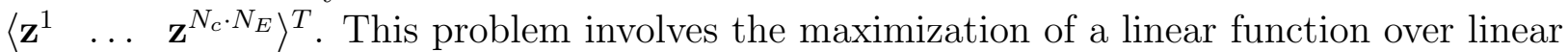
equality constraints and conic constraints which is a standard SOCP problem. The total size of the conic constraints depends on the chosen strength criterion symbolized by $\mathcal{C}$.

\section{Some comments about the shear locking effect}

\subsection{Shear locking effect in displacement/velocity-based finite elements}

The shear-locking effect corresponds to a lack of robustness of some displacement-based (or velocity-based in the context of yield design/limit analysis) thick plate finite elements in the thin plate limit $[22,23]$. Usually, the finite element solution corresponding to a fixed mesh size strongly deteriorates when the plate thickness tends to zero, due to the presence of spurious shear strains. In order to obtain a finite element solution close to the exact thin plate solution, the mesh size has to be increasingly smaller for increasingly smaller values of the plate thickness. Mathematically speaking, the convergence of the finite element solution is non-uniform with respect to the plate thickness $t$.

The source of the shear-locking phenomenon can be found in a mismatch between the discrete thick plate functional space and the functional space corresponding to the thin plate solution. Indeed, the thick plate kinematics consists of a transversal velocity field $w$ and a rotation velocity vector $\beta$. When using, for instance, a continuous piecewise-linear interpolation for the velocity field $w$ and the rotation field $\underline{\beta}$, the Love-Kirchhoff kinematic constraint $\underline{\nabla w}-\underline{\beta}=\underline{0}$ must hold true at the thin plate limit inside each element, so that $\underline{\beta}$ must be in fact constant in the element. Since it is also continuous, it has to be constant 
over the whole domain, $w$ being therefore linear over the whole domain. In the case of non-trivial boundary conditions prescribing zero value of $w$ and/or $\beta$ on some part of the plate boundary, the only possible choice satisfying the Love-Kirchhoff condition is $w=0$ and $\beta=0$. This interpolation will then lead to a convergence of the FE solution to the zero field when $t$ approaches zero instead of converging to the true solution.

Various numerical strategies have been proposed to overcome this shear-locking effect, relying mainly on some kind of relaxation of the strain compatibility equations. Since the present work addresses the development of thick plate equilibrium finite elements in the context of a yield design static approach, it seems valuable to investigate the question of the possible occurence of a shear-locking effect for equilibrium finite elements.

\subsection{The case of equilibrium finite elements}

A first answer can be obtained when looking at the functional spaces used in the static approach. For thick plates, the bending moment field belongs to the functional space of symmetric tensors which are $L^{\infty}(\Omega)$, i.e. bounded over the domain (finite resistance to bending) and the divergence of which (the shear vector) is also bounded. In the thin plate limit, the only difference comes from the fact that the shear strength is now unbounded. So that, the bending moment still belongs to $L^{\infty}(\Omega)$, while its divergence can now be unbounded. Hence, the thin plate functional space is here larger than the thick plate functional space. This is the main difference compared to the kinematic approach for which, due to the LoveKirchhoff kinematic condition, the thin plate functional space is smaller than the thick plate functional space.

Therefore, when using a discrete interpolation of the bending moment in the thick plate case, it is still valid in a thin plate context, irrespectively of the thickness value. It can be expected that no shear locking i.e. no deterioration of the solution, with respect to the thickness, will occur when using equilibrium finite elements.

\section{Illustrative examples}

\subsection{Square plate under uniformly distributed pressure}

The problem of a square plate of side length $L=1$ under uniformly distributed pressure $p_{D}=p$ and various support conditions is first analyzed (figure 7). Owing to symmetry conditions, only the bottom-left quarter of the plate has been discretized with two different unstructured meshes : a coarse mesh with 3 elements per half-side $\left(N_{E}=24\right.$ elements) and a fine mesh with 15 elements per half-side $\left(N_{E}=532\right.$ elements).

The boundary conditions ensuring symmetry at $x=L / 2$ and $y=L / 2$ are $M_{n t}=0$ and $V_{n}=0$. For the plate edges $x=0$ and $y=0$, either simple supports $\left(M_{n n}=0\right)$ or clamped supports (no condition on the static variables) are investigated. The slenderness ratio of the plate is defined as $\beta=L / t$. Unless otherwise specified, the number of checking points has been taken equal to $N_{c}=10$. 

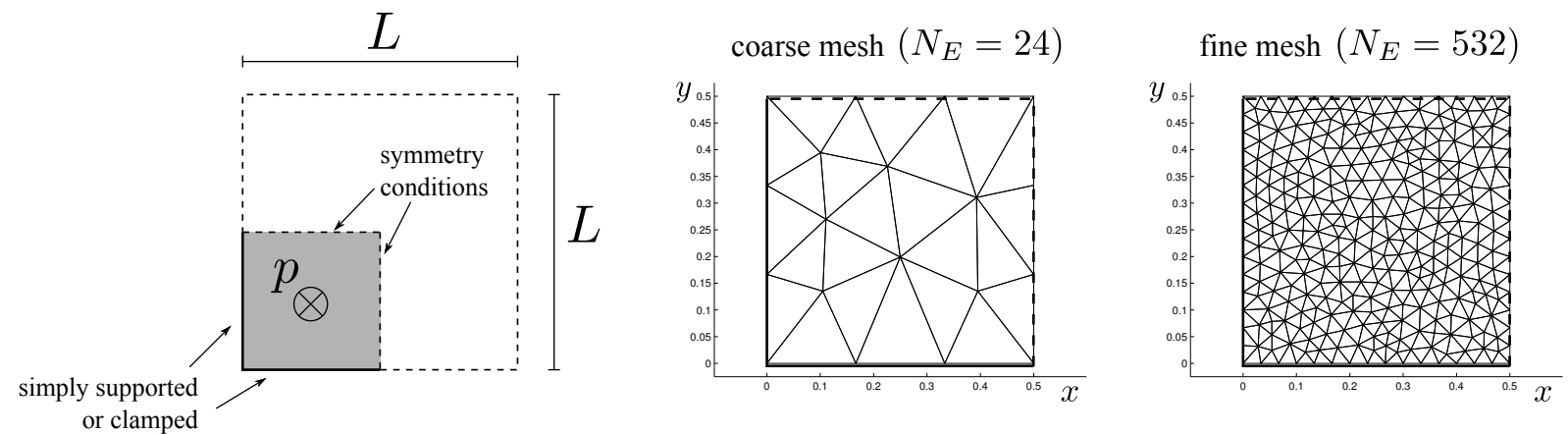

Figure 7: Square plate problem under uniformly distributed pressure

\begin{tabular}{|c||c|c|c||c|}
\hline Boundary conditions & $N_{E}=24$ & $N_{E}=532$ & $N_{E}=2172$ & Upper bounds \\
\hline \multirow{2}{*}{ simply supported } & 24.885 & 25.018 & 25.018 & 25.033 \\
& $(0.59 \%)$ & $(0.06 \%)$ & $(0.06 \%)$ & \\
\hline \multirow{2}{*}{ clamped } & $\begin{array}{c}43.442 \\
(1.71 \%)\end{array}$ & $\begin{array}{c}44.075 \\
(0.27 \%)\end{array}$ & $\begin{array}{c}44.106 \\
(0.20 \%)\end{array}$ & \multirow{2}{*}{44.196} \\
\hline
\end{tabular}

Table 2: Comparison of the lower bound estimates obtained with the infinite shear strength criterion with Love-Kirchhoff thin plate upper bound estimates. The ultimate load estimates are given in terms of the ultimate load factor $\lambda=p L^{2} / M_{0}$, the number in parentheses indicates the relative error in percent compared to the upper bound estimate.

\subsubsection{Thin plate solution with the infinite shear strength criterion.}

First, the criterion with infinite shear strength is adopted. It can be easily seen that the ultimate load $p^{+}$is proportional to $M_{0} / L^{2}=\left(\sigma_{0} / 4\right)(t / L)^{2}$ and should correspond to the ultimate load obtained with a thin plate model. In table 2 , the lower bounds obtained with different meshes are compared to the upper bound ones obtained in [13, 24] by a kinematical approach with a Love-Kirchhoff thin plate model and a majoration procedure to ensure a strict upper bound status. The lower bound estimates are very close to the upper bound estimates which enable to bracket the exact ultimate load of these two problems within less than $0.2 \%$. In the following, the lower bound estimates obtained with a very fine mesh $\left(N_{E}=2172\right.$ elements) will serve as reference values for the thin plate solutions and will be noted $p(\beta=\infty)$.

\subsubsection{Influence of the slenderness ratio on the ultimate load.}

Secondly, the evolution of the lower bound estimate with respect to the slenderness ratio is investigated. Results are presented in figure 8 for simple supports and in figure 9 for clamped supports. The distinction is made between the criterion with or without shearbending interaction. Clearly, it can be observed that for $\beta \geq 10$ (and $\beta \geq 50$ for the clamped case) the lower bound estimates are close to the thin plate solution by less than $1 \%$. It is also worth noting that, even if the solutions obtained with both strength criteria coincide for $\beta$ close to 1 , they produce significantly different results in the regions around $\beta=5$ for simple supports and $\beta=10$ for clamped supports, when the solution exhibits a 


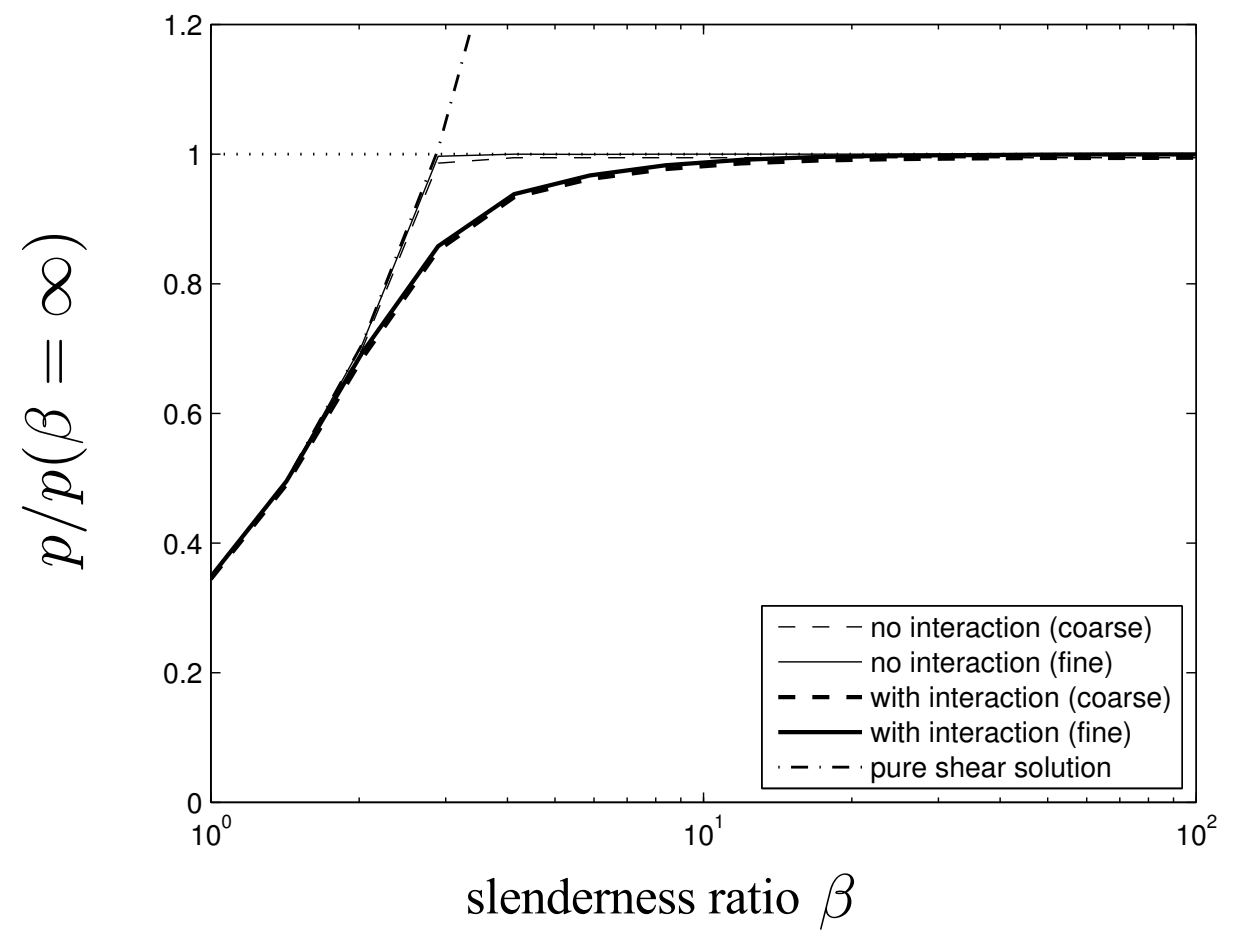

Figure 8: Evolution of the lower bound estimate for a simply supported plate with respect to the slenderness ratio $\beta$. Results are normalized by the thin plate reference solution $p(\beta=\infty)=25.018 M_{0} / L^{2}$. 


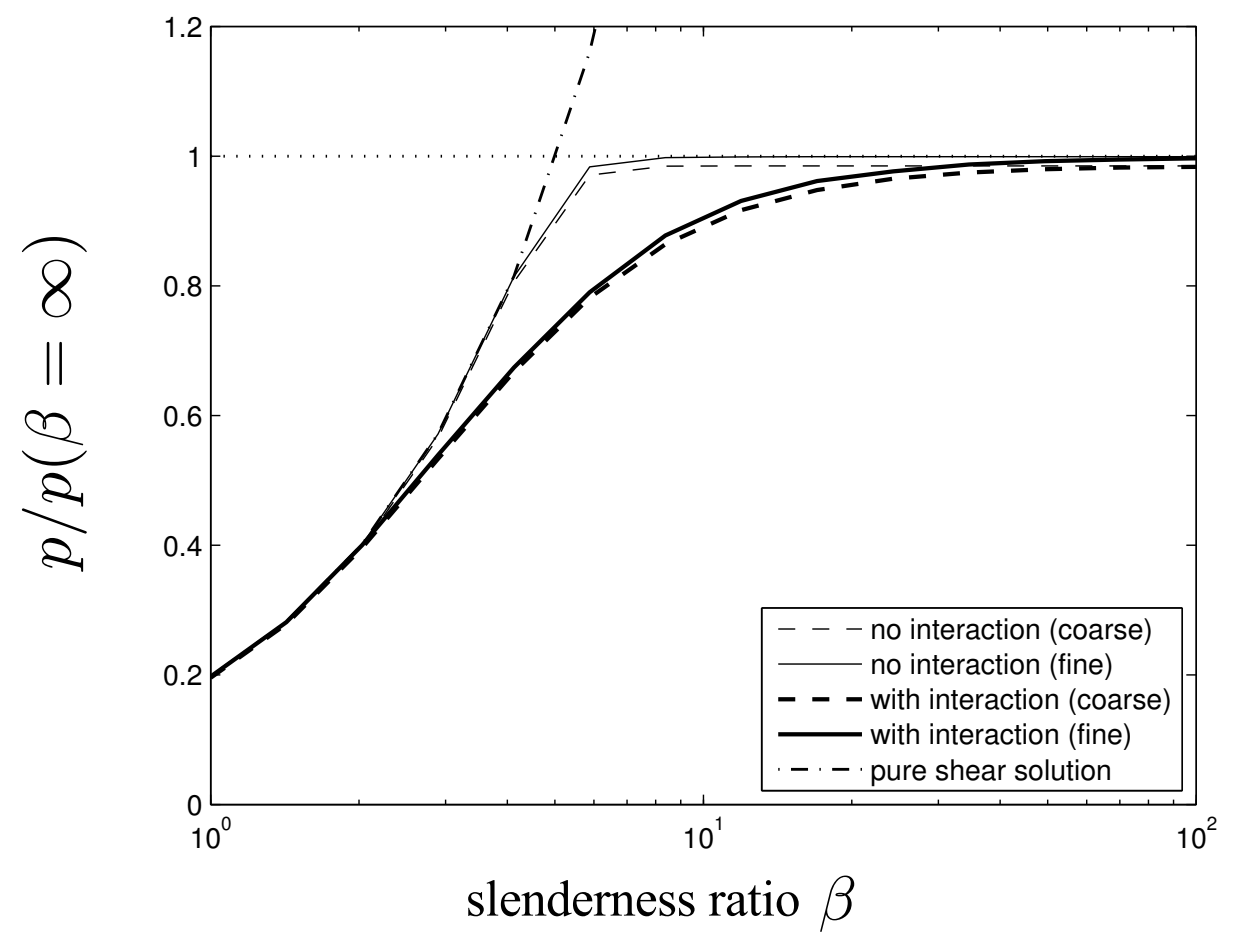

Figure 9: Evolution of the lower bound estimate for a clamped plate with respect to the slenderness ratio $\beta$. Results are normalized by the thin plate reference solution $p(\beta=\infty)=44.106 M_{0} / L^{2}$. 
combination of shear and bending.

Moreover, the ultimate load obtained in the case of a plate resisting only to shear forces (pure shear solution) has also been represented. This solution is given by $[25,26]$

$$
\frac{p L^{2}}{M_{0}}=\frac{4}{\sqrt{3}} \frac{4-\pi}{2-\sqrt{\pi}} \frac{L}{t}
$$

and is independent from the boundary conditions. As expected, the computed estimates are a lower bound of this exact solution and agree quite well with the results in the thick plate limit. Thus, for $\beta=1$, a collapse load of $p L^{2} / M_{0}=8.707$ is obtained for both type of supports, whereas the pure shear solution yields $p L^{2} / M_{0} \approx 8.712$. Despite an error being less than $0.1 \%$, the slight difference with this analytical result can be understood by recalling that the collapse mechanism of the square plate perfect shear solution corresponds to a uniform transversal translation of a region consisting of a square, the corners of which are rounded by circles of radius $\rho=\frac{2-\sqrt{\pi}}{4-\pi} L$, the other part of the plate remaining fixed. The previous results could, therefore, be improved by adapting the mesh along this region to reproduce the optimal fields more accurately. Finally, it is also worth noting that the estimates obtained with the no shear-bending interaction strength criterion are very close to the upper bound obtained by combining the pure shear solution and the thin plate solution (pure bending),

$$
p=\min \left(\frac{4}{\sqrt{3}} \frac{4-\pi}{2-\sqrt{\pi}} \frac{M_{0}}{t L}, p(\beta=\infty)\right)
$$

\subsubsection{Static fields and influence of the shear and bending parts of the strength criterion.}

By way of an example, the different components of the static fields, solution of the simply supported plate problem with $\beta=2.5$ with a shear-bending interaction criterion, are represented in figure 10 for the most refined mesh. In order to investigate the influence of both shear and bending to the satisfaction of the strength criterion, we define the following quantities :

$$
s_{M}=\frac{\|\underline{\underline{M}}\|_{v M}}{M_{0}} \quad \text { and } \quad s_{V}=\frac{\|\underline{V}\|}{V_{0}}
$$

so that the strength criterion with no interaction can be expressed as $s_{M} \leq 1, s_{V} \leq 1$ and the strength criterion with interaction is given by $\sqrt{s_{M}^{2}+s_{V}^{2}} \leq 1$. The local variation of $s_{M}$ and $s_{V}$ are represented in figure 11 for the previous problem. It can clearly be observed that the bending part of the criterion is saturated at the plate center and in the corners, whereas the shear part of the criterion is saturated on the plate edges away from the corners.

Let also define $\overline{s_{M}}$ (resp. $\overline{s_{V}}$ ) as the spatial average of $s_{M}$ (resp. $s_{V}$ ) over the whole plate which may represent a global indicator of a pure-bending, pure-shear or mixed shear-bending problem. The evolution of these two quantities with respect to $\beta$ for simply supported boundaries and the two shear-bending criteria is represented in figure 12. Here again, these results suggest that for $\beta$ being close to 1 , the problem is shear-dominated as expected, whereas it is bending-dominated for $\beta \gg 1$. For intermediate values of $\beta$, the behavior is different given the presence or the absence of interaction between shear and bending. 


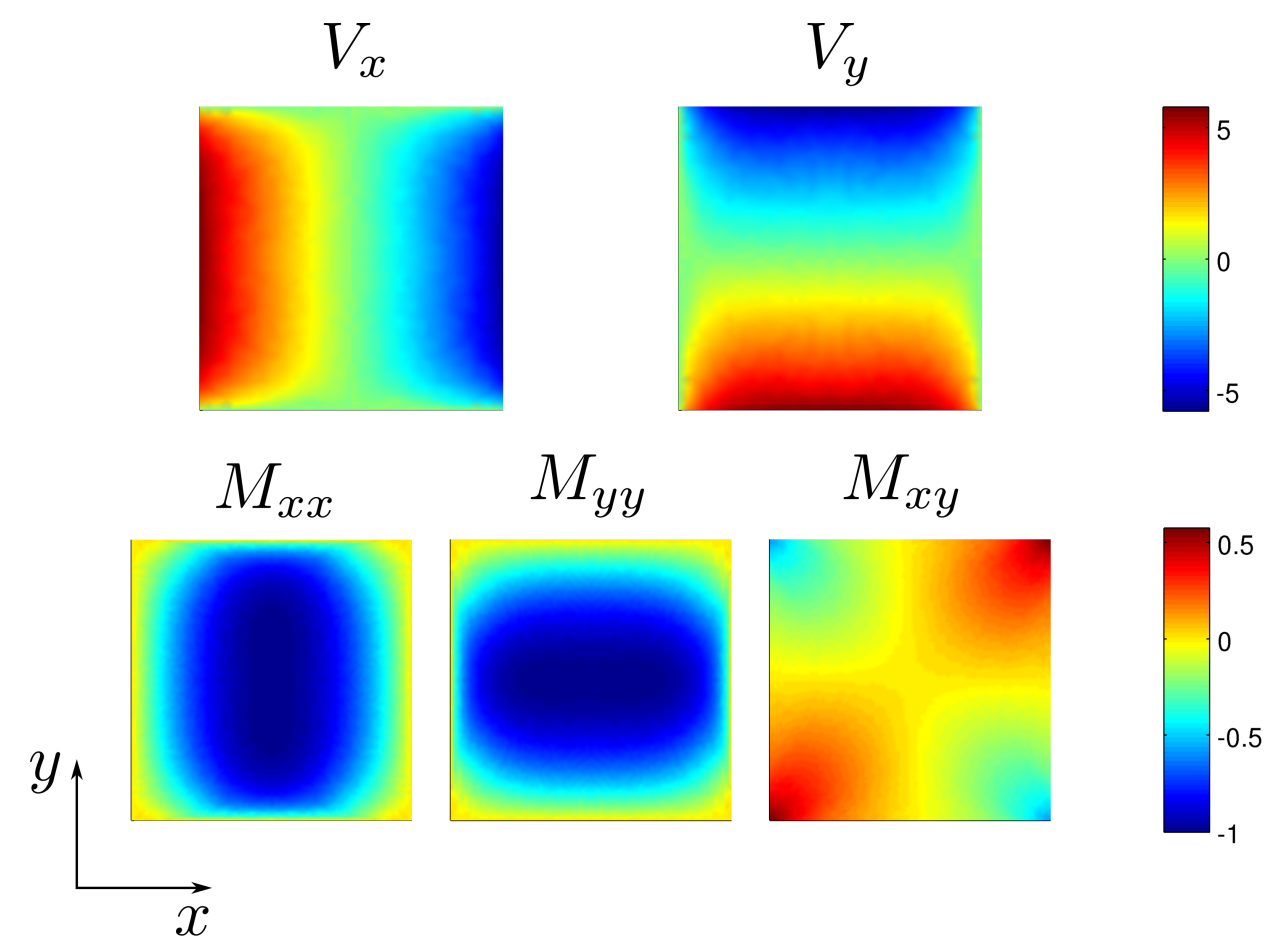

Figure 10: Spatial variation of the static field components solution of the simply supported plate problem with $\beta=2.5$ and a shear-bending interaction criterion $\left(M_{0}=1, V_{0}=5.8\right)$

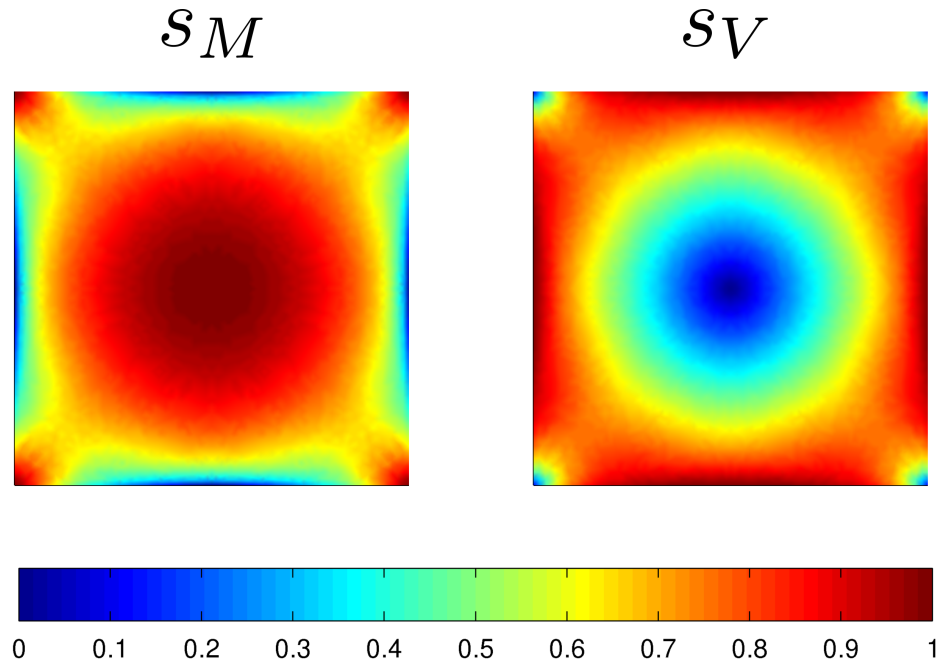

Figure 11: Spatial variation of strength criterion bending part $s_{M}$ and shear part $s_{V}$ 


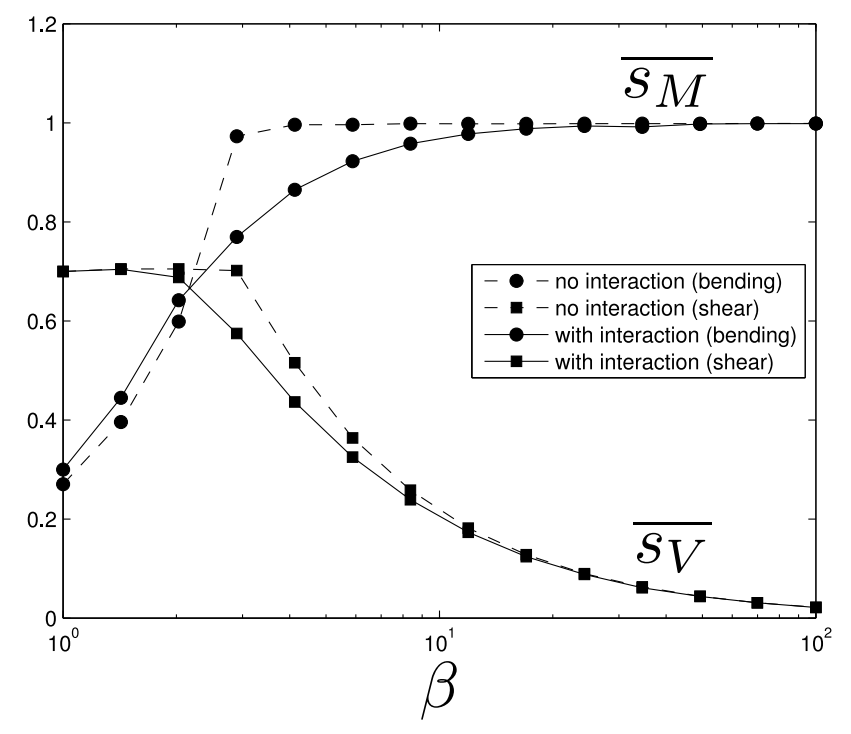

Figure 12: Evolution of $\overline{s_{M}}$ and $\overline{s_{V}}$ with respect to $\beta$ for the simply supported plate problem

As regards the criterion without interaction, the bending part of the criterion is almost saturated in the whole plate for $\beta \geq 3$ whereas the shear part is decreasing with higher values of $\beta$.

\subsubsection{Investigation of the influence of the number of checking points.}

It is also of interest to study the influence of the number of checking points on the lower bound estimates. In table 3, the value of the lower bound estimates are given for the simply supported case with a shear-bending interaction criterion for different types of meshes and different values of $\beta$ and $N_{c}$.

First, the relative difference between $N_{c}=6$ and $N_{c}=10$ are always less than $1 \%$ which ensures that the produced values are close to the converged values. Secondly, it can be observed that the difference increases with increasing values of $\beta$, which can be attributed to the fact that for large values of $\beta$ the problem is bending dominated and it is the bending part of the criterion which is influenced by the number of checking points. Indeed, for $\beta=1$, the ultimate load value is strongly dominated by the shear criterion which is always exactly satisfied in the whole element if it is satisfied for all three corners of the triangle. Hence, there is no modification in taking $N_{c}=6,7$ or 10 in this case. Finally, we also observe that the relative differences at fixed values of $\beta$ decrease with mesh refinement. Obviously, this can be attributed to the fact that with smaller elements, the variation of the local bending moment field are less important and it is, then, more difficult to violate the strength criterion between checking points inside an element.

Therefore, it can be safely concluded that, with a sufficiently fine mesh, the obtained ultimate load estimates are pseudo lower bounds of very good accuracy, although there is no strict assurance that the criterion is not locally violated within an element. 


\begin{tabular}{|c|c|c|c|c|}
\hline & mesh type & $N_{c}=6$ & $N_{c}=7$ & $N_{c}=10$ \\
\hline \multirow[t]{2}{*}{$\beta=1$} & coarse & $\begin{array}{c}8.6175 \\
(0 \%)\end{array}$ & $\begin{array}{c}8.6175 \\
(0 \%)\end{array}$ & $\begin{array}{c}8.6175 \\
(-)\end{array}$ \\
\hline & fine & $\begin{array}{c}8.7056 \\
(0 \%)\end{array}$ & $\begin{array}{c}8.7056 \\
(0 \%)\end{array}$ & $\begin{array}{c}8.7056 \\
(-)\end{array}$ \\
\hline \multirow[t]{2}{*}{$\beta=10$} & coarse & $\begin{array}{l}24.7048 \\
(0.54 \%)\end{array}$ & $\begin{array}{l}24.6032 \\
(0.13 \%)\end{array}$ & $\begin{array}{c}24.5718 \\
(-)\end{array}$ \\
\hline & fine & $\begin{array}{l}24.7113 \\
(0.01 \%)\end{array}$ & $\begin{array}{l}24.7100 \\
(0.00 \%)\end{array}$ & $\begin{array}{c}24.7098 \\
(-)\end{array}$ \\
\hline \multirow[t]{2}{*}{$\beta=100$} & coarse & $\begin{array}{l}25.0471 \\
(0.67 \%)\end{array}$ & $\begin{array}{l}24.9159 \\
(0.14 \%)\end{array}$ & $\begin{array}{c}24.8814 \\
(-)\end{array}$ \\
\hline & fine & $\begin{array}{l}25.0159 \\
(0.00 \%)\end{array}$ & $\begin{array}{l}25.0150 \\
(0.00 \%)\end{array}$ & $\begin{array}{c}25.0148 \\
(-)\end{array}$ \\
\hline
\end{tabular}

Table 3: Influence of the number of checking points on the lower bound estimates $\lambda(\beta)=p(\beta) L^{2} / M_{0}$. The number in parentheses indicate relative error compared to the corresponding value obtained for $N_{c}=10$.

\subsubsection{Comparison of soft/hard simple supports and boundary layer effect.}

The comparison of the Love-Kirchhoff and Reissner-Mindlin plate models has already been widely discussed in the literature, but mostly in the case of linear elasticity and with reference to three-dimensional solutions. One interesting aspect of the comparison between both models concerns the boundary conditions. The Reissner-Mindlin plate model is, indeed, able to distinguish the case of so-called "hard" and "soft" supports, i.e. whenever the displacement is fixed, the rotation around the normal to the boundary is either fixed ("hard" supports) or free ("soft" supports). For simply supported stress-like boundary conditions, this corresponds to imposing only $M_{n n}=0$ (hard supports) or $M_{n n}=M_{n t}=0$ (soft supports). One main question concerns the behavior of those boundary conditions in the limit range of a very small thickness, since in a Love-Kirchhoff thin plate model, only hard supports are admissible due to the enforced relation between the displacement gradient and the local rotation. In the framework of linear elasticity, it has been shown that, in some cases, the Reissner-Mindlin solution converges to the Love-Kirchhoff solution in the thin plate limit except in a boundary layer zone which can appear along the plate edges $[27,28]$. It is, therefore, interesting to investigate this question in the framework of yield design and without any comparison to three-dimensional solutions.

For this purpose, the simply supported square plate problem with an infinite shear strength criterion and hard or soft supports has been investigated. The ultimate load estimates obtained with different values of the mesh size $h=L / 2 / M_{E}, M_{E}$ being the number of elements used to mesh the plate half-sides, have been compared to the reference value of the simply supported plate problem with hard supports, consistent with a Love-Kirchhoff model, $p_{L K}^{*}=25.018 M_{0} / L^{2}$. Three different meshes have been used : a uniform unstructured mesh (mesh A) and two meshes specifically devised to capture a boundary layer along the plate simply supported edges (figure 13). The first one is adapted along a boundary 


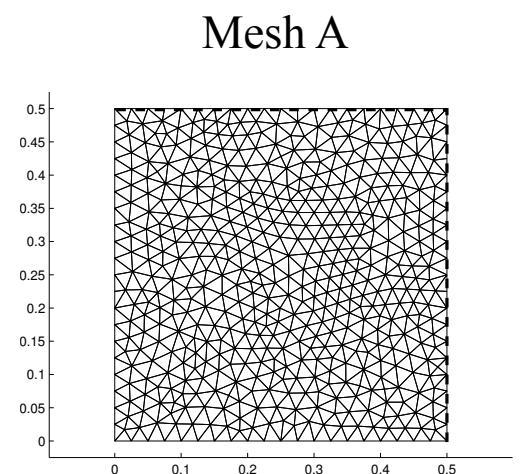

no boundary layer
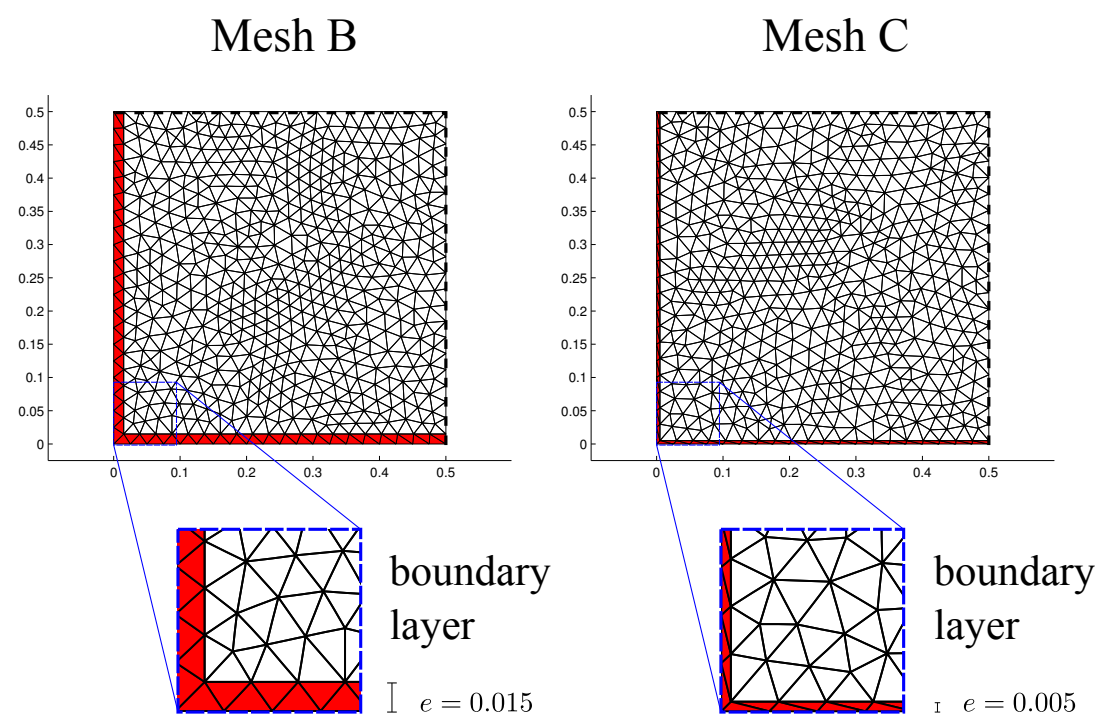

Figure 13: Three types of meshes used to compare the influence of hard or soft supports. Represented meshes correspond to $M_{E}=20$ elements per plate half-side $(h=0.025)$.

layer of width $e=0.015$ (mesh B), whereas the second one is adapted along a boundary layer of width $e=0.005$ (mesh $\mathrm{C}$ ). Note that there is only one element in the boundary layer width direction.

The evolution of the relative error (defined as $1-p / p_{L K}^{*}$ ) with respect to the mesh size $h$ has been represented in figure 14. First, it has been observed that, in the case of hard supports $\left(M_{n n}=0\right.$ only), the ultimate loads obtained at a fixed mesh size do not depend on the type of mesh (unstructured or adapted) which has been used. The results presented in figure 14 for the hard support case (crosses) correspond, therefore, only to an unstructured mesh of type A. Hence, one may conclude that the solution corresponding to the hard support case do not present any rapidly varying feature along the plate edges.

On the contrary, in the case of soft supports $\left(M_{n n}=M_{n t}=0\right)$, the results are quite dependent on the mesh employed. It can first be observed that the convergence rate is in $O\left(h^{0.9}\right)$ for mesh A whereas the convergence rate for hard supports is sensibly faster as it varies as $O\left(h^{2.6}\right)$. As regards meshes adapted along a boundary layer, the convergence rate is close to that of the hard support case for the largest values of $h$. Thus, it seems that the solution corresponding to the simple support case presents a rapidly varying feature along the plate edges, since it is better approximated by boundary layer-adapted meshes. Besides, at a certain critical mesh size, convergence is stopped which can be explained by the fact that the solution can no longer be improved with mesh refinement in this region because the typical element size is fixed by the boundary layer width $e$. It is also clear that this effect is delayed for a smaller width $e$.

Finally, the analysis of the static fields in this boundary layer shows a distribution of almost infinite shear forces which counterbalance the transition of the $M_{n t}$ component from 0 to a non-zero value through the boundary layer. 


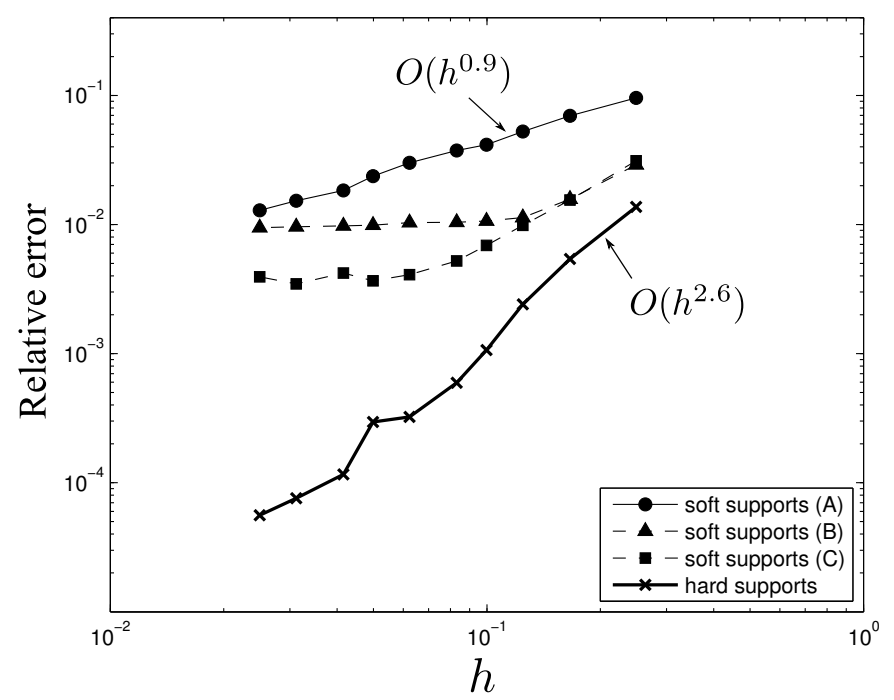

Figure 14: Relative error convergence of the simply supported plate problem with hard/soft supports for different types of meshes : circles = uniform unstructured mesh (type A), triangles = mesh adapted along a boundary layer of width $e=0.015$ (type B), squares = mesh adapted along a boundary layer of width $e=0.005$ (type C)

Hence, it can be concluded that the hard support problem converges normally to the thin plate limit value, whereas the soft support problem converges to the same value, but also exhibits a boundary layer effect near the plate supports due to the additional constraint $M_{n t}=0$, as is the case in the context of elasticity.

\subsection{Circular plate examples}

In this second example, the ultimate load of simply supported and clamped circular plates of radius $R$ is investigated under a uniformly distributed load $p$. These problems have been treated in the work of Capsoni and Vicente da Silva [17] using an upper bound kinematic approach based on a fully 3D Reissner-Mindlin element. In order to avoid shear locking in the thin plate limit, they proposed to relax the strain compatibility equation using a B-bar method. For this reason, the produced collapse load estimates are not exact upper bounds of the true collapse load but only pseudo-upper bounds. Besides, the maximum resisting power is obtained by integrating the expression corresponding to the $3 \mathrm{D}$ von Mises criterion through the thickness. Therefore, their criterion is slightly different from the criterion with shear-bending interaction that is considered here. In fact, the latter is obtained by using only a one point quadrature in the integral through the plate thickness [11]. However, their results are still very interesting to assess the efficiency of the present static approach in the case of a strength criterion with shear-bending interaction.

For all computations, only a quarter of the circular plate has been meshed using an unstructured mesh consisting of 726 elements. For both boundary conditions, the lower bound estimates obtained for different slenderness ratio $\beta=R / t$ have been compared to the corre- 


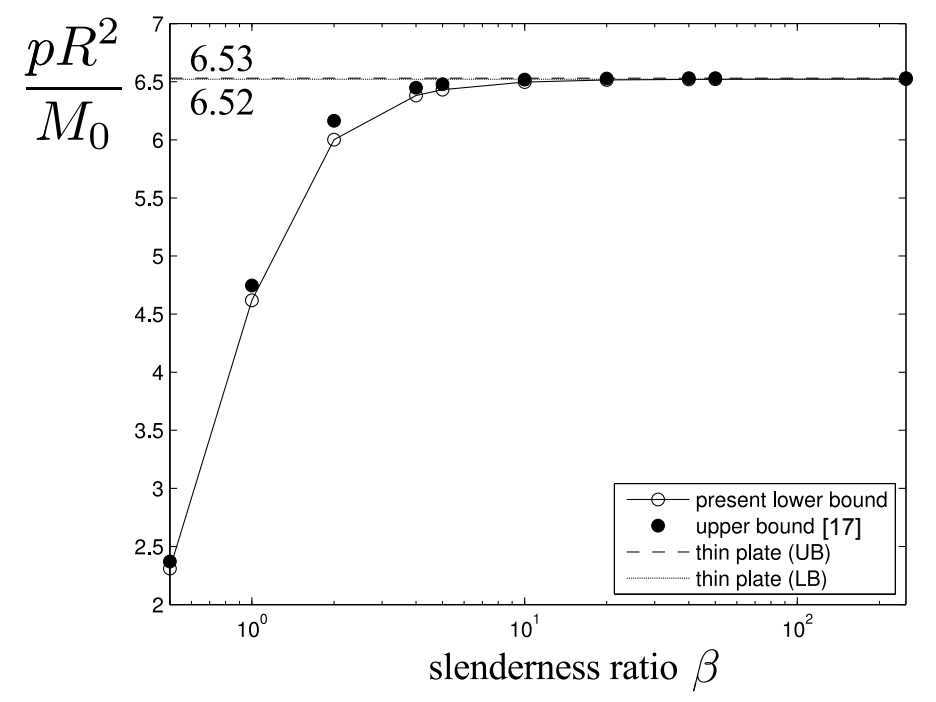

Figure 15: Collapse load of a simply supported circular plate with different slenderness ratios

sponding (pseudo)-upper bounds obtained with the finest mesh considered in [17]. Besides, collapse loads corresponding to a thin plate model have also been represented. Thin plate lower bounds have been computed using the present method with the infinite shear strength criterion whereas upper bounds have been computed using the H3 finite element kinematic approach developed in [13, 24].

As regards the simply supported case (figure 15), it can be observed that the results obtained from the present lower bound approach and those obtained from [17] agree very well for the whole considered range of slenderness ratio. In the thin plate limit $\beta \rightarrow \infty$, the computed values converge, as expected, to the solution obtained with a thin plate model, the remaining discrepancy being around $0.1 \%$. Moreover, in the thick plate limit for $\beta=0.5$, a collapse load of $p R^{2} / M_{0}=2.309$ is obtained. This result has to be compared to the pure shear solution,

$$
\frac{p R^{2}}{M_{0}}=\frac{4}{\sqrt{3}} \frac{2 R}{t} \approx 2.3094
$$

which is independent from the boundary conditions.

As regards the clamped case (figure 16), it can first be noted that the same value as the simply supported case is obtained for $\beta=0.5$ with our method. For higher values of $\beta$ a larger discrepancy than in the simply supported case can be observed between our lower bound and Capsoni and Vicente da Silva's pseudo-upper bound. Considering that our lower bound yields the correct value in the thin plate limit (the error being still around $0.1 \%$ ), the difference can be attributed to the lack of performance of the upper bound finite element used in [17] for this problem. This can be explained by the fact that, contrary to the thin plate finite element of $[13,24]$, they used a finite element which cannot account for rotation discontinuities along the clamped boundary, thus requiring the use a very fine mesh near 


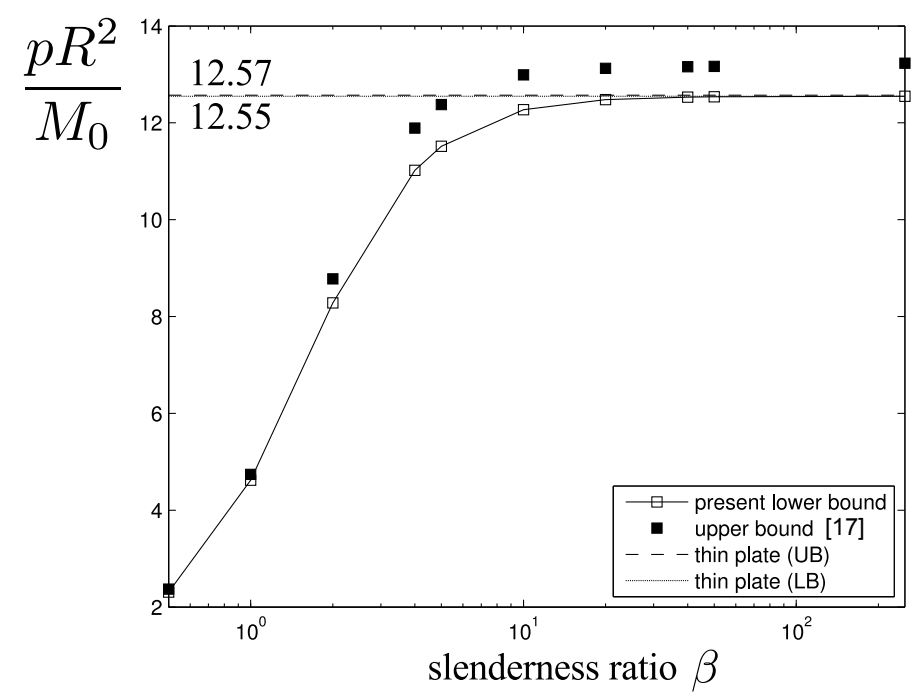

Figure 16: Collapse load of a clamped circular plate with different slenderness ratios

the clamped boundary in order to improve the quality of the upper bound.

\subsection{L-shaped plate}

The next examples consider a L-shaped plate under uniformly distributed loading and boundary conditions as shown in figure 17. Three different structured mesh of the type represented in figure 17 are considered with a total number of elements taking the values $N_{E}=150,600$ and 2400. Here again, different slenderness ratios are considered with the ellipsoid-type interaction criterion and compared to the upper bounds values obtained by Le [18] for the same problem. Limit loads are given in a dimensionless form, that is, as $\lambda^{+}=\left(q^{+} L^{2}\right) / M_{0}$. The obtained lower bounds are given in table 4 where the line $\beta \rightarrow \infty$ corresponds to the thin plate limit with the unlimited shear force criterion.

The results are seen to be quite close to the upper bounds computed in [18] in which an adaptive refinement of the mesh has been used to produce those values. Here, it can be seen that the lower bound estimates converge very quickly when refining the mesh and that there is no sign of deterioration of the convergence in the thin plate limit, which is a further evidence indicating that the proposed elements seem to be free from any shear-locking.

\subsection{Rectangular plate with eccentric square cutout}

In this last example, the problem of a rectangular plate with an eccentric square cutout is investigated. This problem has been treated in [18] by a kinematic approach for two different slenderness ratios. The plate is subjected to a uniformly distributed loading of magnitude $q$ and all external and internal edges are simply supported. The geometry is described in figure 18 .

Lower bound estimates have been obtained for three different meshes and two different slenderness ratios $\beta=1$ and $\beta=100$. In this example, the slenderness ratio is $\beta=L / t$ with 


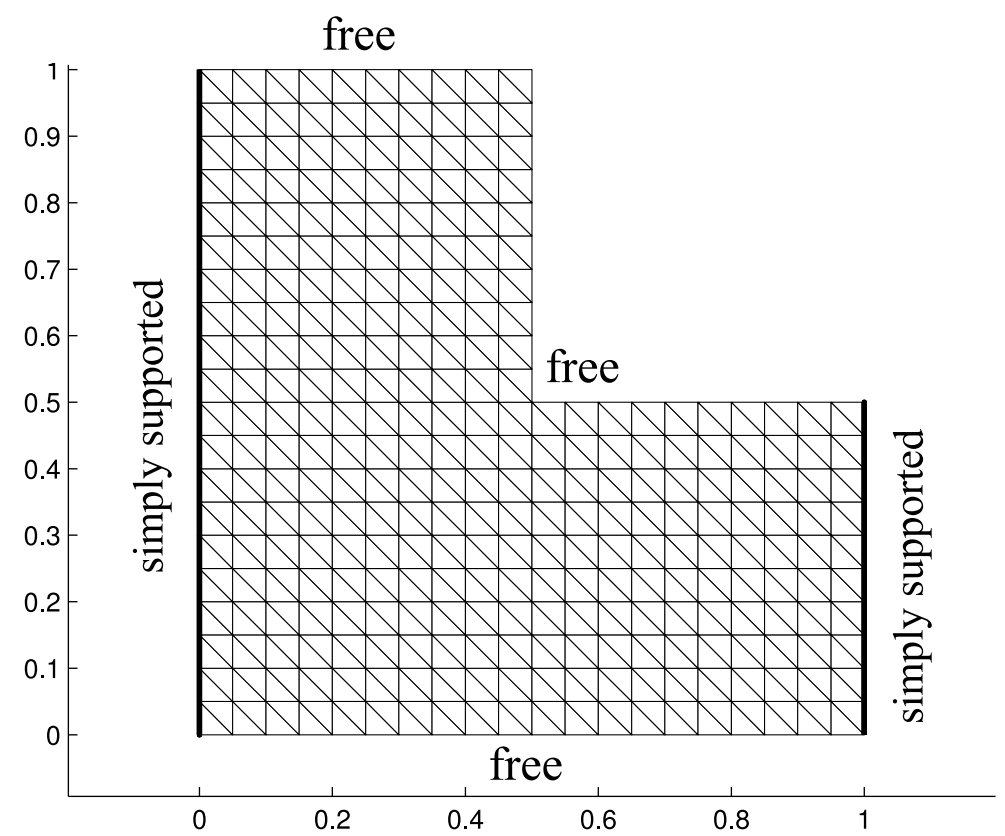

Figure 17: L-shaped plate problem meshed with $N_{E}=600$ elements

\begin{tabular}{ccccc}
\hline$\lambda^{+}$ & $N_{E}=150$ el. & $N_{E}=600$ el. & $N_{E}=2400$ el. & Upper bound [18] \\
\hline$\beta=1$ & 3.7 & 3.7 & 3.7 & 3.73 \\
$\beta=2$ & 5.67 & 5.68 & 5.69 & 5.8 \\
$\beta=4$ & 5.87 & 5.88 & 5.89 & 5.98 \\
$\beta=8$ & 5.98 & 6 & 6 & 6.08 \\
$\beta=10$ & 6 & 6.02 & 6.03 & 6.1 \\
$\beta=20$ & 6.03 & 6.07 & 6.08 & 6.14 \\
$\beta=40$ & 6.04 & 6.08 & 6.1 & 6.16 \\
$\beta=80$ & 6.05 & 6.09 & 6.11 & 6.18 \\
$\beta=100$ & 6.05 & 6.09 & 6.11 & 6.18 \\
\hline$\beta \rightarrow \infty$ & 6.05 & 6.09 & 6.11 & - \\
\hline
\end{tabular}

Table 4: Lower bound estimates for the L-shaped plate for three different meshes. 


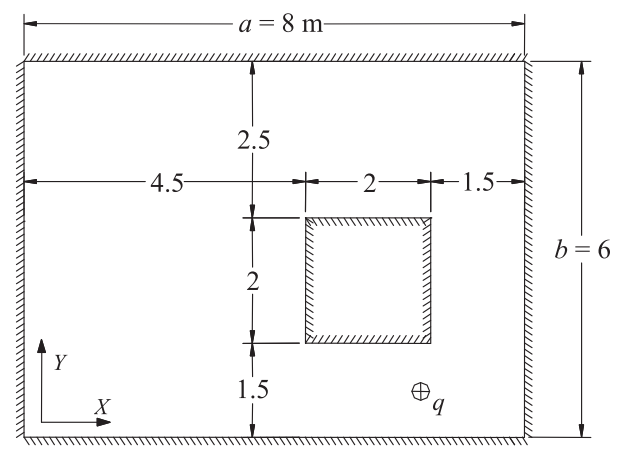

(a) Geometry and boundary condition $[18]$

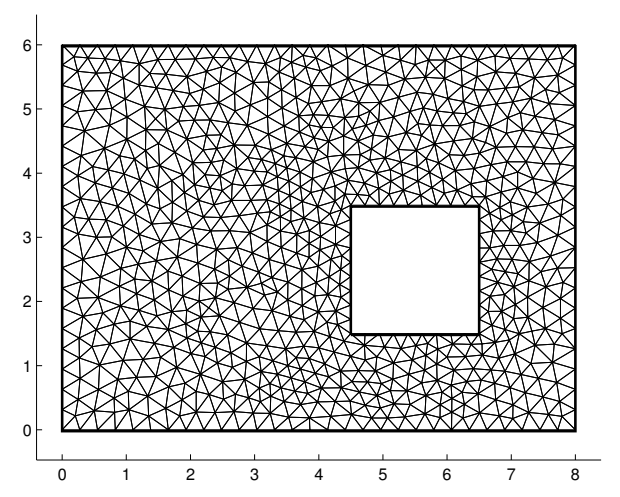

(b) Mesh corresponding to $N_{E}=1464$ elements

Figure 18: Rectangular plate with an eccentric square hole

\begin{tabular}{ccccc}
\hline$\lambda^{+}$ & $N_{E}=314$ el. & $N_{E}=1464$ el. & $N_{E}=3590$ el. & Upper bound $[18]$ \\
\hline$\beta=1$ & 13.30 & 13.35 & 13.37 & 13.69 \\
$\beta=100$ & 48.42 & 48.78 & 48.82 & 49.46 \\
\hline
\end{tabular}

Table 5: Lower bound estimates for the rectangular plate with eccentric square cutout for three different meshes.

$L=b=6 \mathrm{~m}$. Limit loads are given in a dimensionless form, that is, as $\lambda^{+}=\left(q^{+} a b\right) / M_{0}$. The obtained lower bounds are given in table 5 and compared to the upper bound estimates obtained in [18].

\section{Conclusion}

The present work formulates a yield design problem for thick plates in bending by taking into account the effect of shear and bending in the plate strength criterion. It seems to be the first time that an equilibrium finite element is proposed to solve the lower bound static approach of this kind of structures. Due to the simplicity of the considered strength criteria, the discretized problem can be cast easily as a standard second-order cone program, taking advantage of the efficiency of dedicated SOCP solvers such as the MOSEK software package. The efficiency of the proposed finite element is validated on different plate examples. In particular, it enables to retrieve the pure shear solutions in the thick plate limit $\beta \sim 1$, as well as the thin plate solutions obtained in previous works with an excellent accuracy (less than 1\%). Contrary to other elements proposed in the available literature, this element seems to be free from any shear locking in the thin plate limit. It has also been shown that, as is the case for elastic problems, a boundary layer effect can occur at the thin plate limit when soft support boundary conditions are used.

Future work will deal with more complex strength criteria than those used in this paper. 
The performance of the proposed method relying, partly, on the efficiency of SOCP solvers, the main difficulty will be to formulate five-dimensional strength criteria which can be expressed as conic constraints without too much effort. One simple extension could be, for example, to use a Johansen square strength criterion for the bending part of the strength criterion.

[1] Sloan SW. Lower bound limit analysis using finite elements and linear programming. International Journal for Numerical and Analytical Methods in Geomechanics 1988; 12(1):61-77.

[2] Pastor J, Turgeman S. Limit analysis in axisymmetrical problems: Numerical determination of complete statical solutions. International Journal of Mechanical Sciences 1982; 24(2):95-117.

[3] Sloan SW. Upper bound limit analysis using finite elements and linear programming. International Journal for Numerical and Analytical Methods in Geomechanics 1989; 13(3):263-282.

[4] Turgeman S, Pastor J. Limit analysis: a linear formulation of the kinematic approach for axisymmetric mechanic problems. International Journal for Numerical and Analytical Methods in Geomechanics 1982; 6(1):109-128.

[5] Johansen K. Yield-line theory. Cement and Concrete Association London, 1962.

[6] Salençon J. Calcul à la rupture et analyse limite. Presses de l'Ecole Nationale des Ponts et Chaussées, 1983.

[7] Save MA, Massonnet CE, de Saxce G. Plastic limit analysis of plates, shells, and disks, vol. 43. North Holland, 1997.

[8] Salençon J. Yield Design. Wiley. com, 2013.

[9] Munro J, Da Fonseca A. Yield line method by finite elements and linear programming. Structural Engineer 1978; 56(2):37-44.

[10] Hodge Jr PG, Belytschko T. Numerical methods for the limit analysis of plates. Journal of Applied Mechanics 1968; 35:796.

[11] Capsoni A, Corradi L. Limit analysis of plates- a finite element formulation. Structural Engineering and Mechanics 1999; 8(4):325-341.

[12] Le CV, Nguyen-Xuan H, Nguyen-Dang H. Upper and lower bound limit analysis of plates using FEM and second-order cone programming. Computers \& Structures 2010; 88(1-2):65-73.

[13] Bleyer J, de Buhan P. On the performance of non-conforming finite elements for the upper bound limit analysis of plates. International Journal for Numerical Methods in Engineering 2013; 94(3):308-330, doi: $10.1002 / n m e .4460$.

[14] Faccioli E, Vitiello E. A finite element, linear programming methods for the limit analysis of thin plates. International Journal for Numerical Methods in Engineering 1973; 5(3):311-325.

[15] Krabbenhoft K, Damkilde L. Lower bound limit analysis of slabs with nonlinear yield criteria. Computers 83 structures 2002; 80(27):2043-2057.

[16] Cecchi A, Milani G, Tralli A. A Reissner-Mindlin limit analysis model for out-of-plane loaded running bond masonry walls. International journal of solids and structures 2007; 44(5):1438-1460.

[17] Capsoni A, Vicente da Silva M. A finite element formulation of Mindlin plates for limit analysis. International Journal for Numerical Methods in Biomedical Engineering 2011; 27(1):143-156.

[18] Le CV. A stabilized discrete shear gap finite element for adaptive limit analysis of MindlinReissner plates. International Journal for Numerical Methods in Engineering 2013; 96:231-246, doi: 10.1002/nme.4560.

[19] Papadopoulos P, Taylor RL. An analysis of inelastic Reissner-Mindlin plates. Finite elements in analysis and design 1991; 10(3):221-233.

[20] Ibrahimbegović A, Frey F. An efficient implementation of stress resultant plasticity in analysis of reissner-mindlin plates. International Journal for Numerical Methods in Engineering 1993; 36(2):303320.

[21] Lubliner J. Plasticity Theory. Dover books on engineering, Dover Publications, 2008.

[22] Arnold DN. Discretization by finite elements of a model parameter dependent problem. Numerische Mathematik 1981; 37(3):405-421. 
[23] Arnold DN, Brezzi F, Falk RS, Marini LD. Locking-free Reissner-Mindlin elements without reduced integration. Computer methods in applied mechanics and engineering 2007; 196(37):3660-3671.

[24] Bleyer J, De Buhan P. A majoration method to obtain true upper bounds for the limit analysis of von Mises plates. CM13 : Proceedings of the International Conference on Computational Mechanics, School of Engineering and Computing Sciences, Durham University (ed.), Durham, UK, 2013; 16-CM13.

[25] Strang G. A minimax problem in plasticity theory. Functional analysis methods in numerical analysis. Springer, 1979; 319-333.

[26] Overton ML. Numerical solution of a model problem from collapse load analysis. Proc. of the sixth int'l. symposium on Computing methods in applied sciences and engineering, VI, North-Holland Publishing Co.: Amsterdam, The Netherlands, The Netherlands, 1985; 421-437.

[27] Arnold DN, Falk RS. The boundary layer for the Reissner-Mindlin plate model. SIAM Journal on Mathematical Analysis 1990; 21(2):281-312.

[28] Arnold DN, Falk RS. Asymptotic analysis of the boundary layer for the Reissner-Mindlin plate model. SIAM Journal on Mathematical Analysis 1996; 27(2):486-514. 